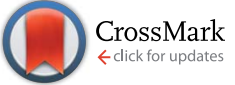

Cite this: RSC Adv., 2017, 7, 15747

\title{
Gradient nanoporous gold: a novel surface- enhanced Raman scattering substrate $\uparrow$
}

\begin{abstract}
Jinglin Huang, ${ }^{a}$ Yansong Liu, ${ }^{a}$ Xiaoshan $\mathrm{He}{ }^{\mathrm{a}}$ Cuilan Tang, ${ }^{\mathrm{ab}}$ Kai Du ${ }^{\mathrm{a}}$ and Zhibing He ${ }^{* a}$
The design and fabrication of surface-enhanced Raman scattering (SERS) substrates with high Raman enhancement, stability, homogeneity and processing compatibility is still one of the most challenging issues in SERS research. In this work, a novel nanoporous gold (NPG) structure that contains a gradient of ligament and pore structures along the thickness direction is designed and simply fabricated by combination of the traditional magnetron sputtering and dealloying technique. This novel nanoporous gold structure (we called it gradient nanoporous gold, GNPG) brings abundant inner structures into conventional nanoporous gold, which can produce remarkable properties for GNPG, such as the surface enhanced Raman scattering. GNPG was demonstrated to have ultrahigh and homogeneous surface enhanced Raman scattering. A simple method was proposed to estimate the relative enhancement factor (EF). The EF of GNPG was estimated to be $\sim 8$-fold higher than conventional NPG, which means that its EF should over $10^{7}$. It is believed that the gradient ligament structure of GNPG plays a key role for its Raman scattering enhancement.
\end{abstract}

Received 23rd December 2016 Accepted 3rd March 2017

DOI: 10.1039/c6ra28591k

rsc.li/rsc-advances

been reported, such as mechanically stamped NPG gratings, ${ }^{13}$ wrinkling NPG films, ${ }^{14}$ NPG disks ${ }^{15-17}$ and patterned NPGs. ${ }^{18}$ These NPG structures generally possess higher SERS enhancement factor (EF) than conventional NPG films. However, some defects exist in these NPGs themselves or their fabrication strategies. The SERS homogeneity of wrinkling NPG films is poor for the random wrinkling distribution. ${ }^{\mathbf{1 4}}$ The stability of NPG disks is poor for the coarsening of nano-sized disks. ${ }^{17}$ The fabrication processes of these NPG structures always tend to be expensive and time-consuming, which limits cost-effectiveness and the potential for mass production. Although mechanically stamped NPG gratings were reported to be fabricated by a onestep process, the processing of the submicron silicon stamps may not be quite easily. ${ }^{13}$

In this work, a novel nanoporous gold structure that contains a gradient of ligaments and pore structures along the thickness direction is designed and simply fabricated by combination of the traditional magnetron sputtering and dealloying technique. We called this kind of nanostructure gradient nanoporous gold (GNPG). The composition, microstructure of GNPGs and their SERS effects for rhodamine 6G (R6G) and crystal violet (CV) molecules are discussed.

\section{Experimental}

${ }^{a}$ Research Center of Laser Fusion, China Academy of Engineering Physics, Mianyang 621900, China

${ }^{b}$ School of Material Science and Engineering, Southwest University of Science and Technology, Mianyang 621010, China

$\dagger$ Electronic supplementary information (ESI) available. See DOI: $10.1039 / \mathrm{c} 6 \mathrm{ra} 28591 \mathrm{k}$

\subsection{Fabrication of gradient nanoporous gold}

GNPGs were simply fabricated by dealloying gradient AuAg alloy precursor films. Fig. 1 shows a schematic diagram of the experimental process. The gradient AuAg alloy precursor films were deposited on $\mathrm{Si}$ substrates by direct-current (dc) 


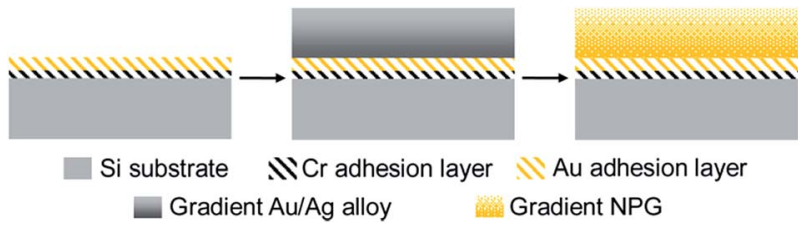

Fig. 1 Schematic illustration of the fabrication process in this study.

magnetron co-sputtering of a $\mathrm{Au}$ target (99.99\% pure) and a $\mathrm{Ag}$ target $(99.9 \%$ pure). Si substrates were washed by acetone, ethanol and deionized water (DI, $18 \mathrm{M} \Omega$ ), successively. The precursor films were sputtered in pure Ar at a working pressure of $0.5 \mathrm{~Pa}$ (3.8 mTorr) at room temperature, while the base pressure was lower than $1.2 \times 10^{-4} \mathrm{~Pa}\left(9 \times 10^{-7}\right.$ Torr $)$. The targets were first pre-sputtered to be cleaned for $150 \mathrm{~s}$, after that a $10 \mathrm{~nm} \mathrm{Cr}$ (Cr target, 99.99\% pure) adhesion layer and $10 \mathrm{~nm}$ $\mathrm{Au}$ adhesion layer was deposited to increase the adhesion strength of AuAg alloy with Si substrate to avoid the fracture of films during dealloying. In order to acquire a gradient content ratio of $\mathrm{Au}$ and $\mathrm{Ag}$ atoms, the power of the targets should be carefully controlled. The power of Ag target was set constant at 50 watts, while the power of Au target was gradually varied. Two sets of precursor films were deposited: (1) $\mathrm{Au}_{15-40} \mathrm{Ag}_{85-60}$ alloy (subscript represents composition changing range), Au target power was varied from 82 to 22 watts; (2) $\mathrm{Au}_{25-40} \mathrm{Ag}_{75-60}$ alloy, $\mathrm{Au}$ target power was varied from 82 to 41 watts. The power of $\mathrm{Au}$ and $\mathrm{Ag}$ target was chosen via the deposition rate of $\mathrm{Au}$ and $\mathrm{Ag}$. The thickness of AuAg alloy layer was deposited approximately to $90 \mathrm{~nm}$. Another two kinds of NPG films $\left(\mathrm{Au}_{15} \mathrm{Ag}_{85}\right.$ and $\mathrm{Au}_{25} \mathrm{Ag}_{75}$ films as the precursors) were prepared for comparison. Dealloying was implemented by dipping AuAg alloy precursor films in $70 \%$ room temperature $\mathrm{HNO}_{3}$ for $5 \mathrm{~min}$ followed by DI rinse and nitrogen dry to form the GNPG films.

\subsection{Characterization}

The composition of GNPG nanostructures was determined by secondary ion mass spectroscopy (SIMS: Hiden EQS), X-ray photoelectron spectroscopy depth profile (XPS: PE PHI-5400) and energy dispersive X-ray spectroscopy (EDS). The time span of each etching time of XPS is $30 \mathrm{~s}$ with the etching depth $\sim 8 \mathrm{~nm}$. The microstructure of GNPGs was examined by a field emission scanning electron microscopy (SEM: ZEISS Merlin VP Compact) operated at an accelerating voltage of $10 \mathrm{kV}$. The surface-enhanced Raman spectroscopy was performed using a micro-Raman spectrometer (Renishaw InVia) with an excitation laser wavelength of $514.5 \mathrm{~nm}$. The laser power was $0.06 \mathrm{~mW}$ and the focused spot size was $1 \mu \mathrm{m}$. The spectra were obtained at random locations for each sample with an integration time of $50 \mathrm{~s}$. Rhodamine $6 \mathrm{G}\left(\mathrm{R} 6 \mathrm{G}, 10^{-7} \mathrm{M}\right)$ and crystal violet $\left(\mathrm{CV}, 10^{-5}\right.$ $\mathrm{M})$ was used as the probe molecules. In order to conduct SERS measurements, the GNPGs on Si substrates were immersed in probe molecules solution for $12 \mathrm{~h}$, rinsed thoroughly with DI, and then dried under blowing $\mathrm{N}_{2}$. The surface area was measured by the capacitance ratio method using $1 \mathrm{M} \mathrm{HClO}_{4}$ as the electrolyte and GNPG samples as the working electrode. ${ }^{\mathbf{1 9 2 0}}$
The $\mathrm{Ag} / \mathrm{AgCl}$ electrode and $\mathrm{Pt}$ wire electrode were used as reference electrode and counter electrode, respectively. The contact area of working electrode with electrolyte was set to be 1 $\mathrm{cm}^{2}$. The cyclic voltammograms was measured using electrochemical workstation (Autolab PGSTAT 302N).

\section{Results and discussion}

\subsection{Composition analysis of precursors and GNPG films}

It is significant to ensure the gradient $\mathrm{AuAg}$ alloy has been deposited as we designed. Therefore, the composition of AuAg alloy precursors was measured by depth dependent composition analysis using SIMS. Fig. 2a shows the variation of $\mathrm{Au}^{+}$ intensity with the etching time in the two kinds of alloy precursors, which can represent the variation of $\mathrm{Au}$ content along the thickness direction. It is clear that the intensity of $\mathrm{Au}^{+}$ increases nearly linearly with the etching time. The intensity of $\mathrm{Au}^{+}$in $\mathrm{Au}_{25-40} \mathrm{Ag}_{75-60}$ is higher than that in $\mathrm{Au}_{15-40} \mathrm{Ag}_{85-60}$ before $200 \mathrm{~s}$, which indicates that the $\mathrm{Au}$ content in $\mathrm{Au}_{25-40} \mathrm{Ag}_{75-60}$ is higher than $\mathrm{Au}_{15-40} \mathrm{Ag}_{85-60}$ near the film surface. While when the etching time increases further, the intensity of $\mathrm{Au}^{+}$is approximately equal for these two precursors. It is not easy to measure the precise element content by SIMS. Thus, the XPS depth profile was further used to demonstrate the gradient composition as shown in Fig. 2b. Clearly, the XPS results appear a similar variation of composition as SIMS. The results of SIMS and XPS demonstrate that the gradient AuAg alloy precursors have been deposited successfully. EDS measurement was made to characterize the total element distributions. The results are listed in Table 1 . Note that the Au content of these two alloy precursors is $\sim 10$ at $\%$ higher than the theoretically average values (that should be 27.5 at\% and 32.5 at\% of these two
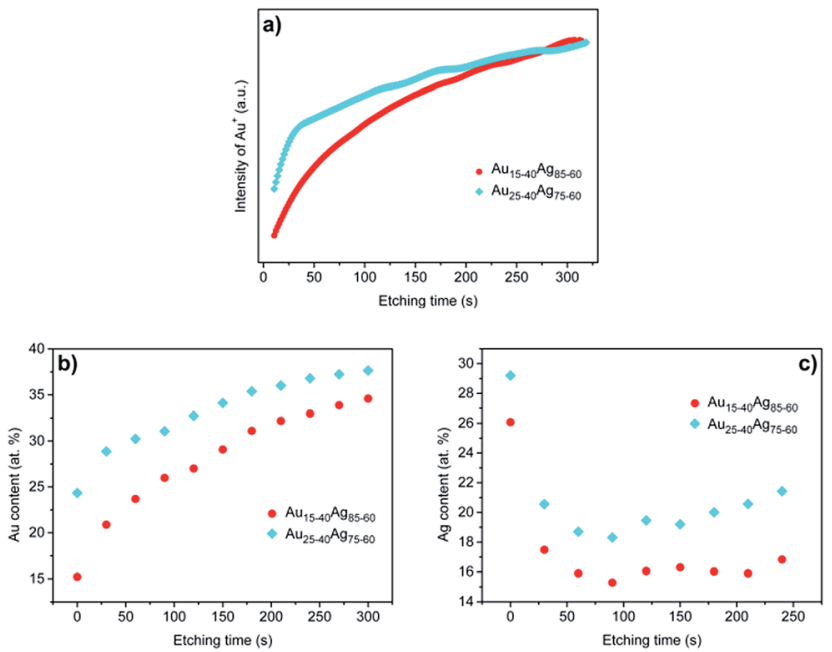

Fig. 2 Variation of composition in alloy film precursors and GNPG films. (a) Variation of the intensity of $\mathrm{Au}^{+}$in precursors with etching time using SIMS. The total etching time of each film is about $330 \mathrm{~s}$ with the corresponding film thickness about $100 \mathrm{~nm}$. (b) Variation of $\mathrm{Au}$ content in precursors by XPS depth profile with 10 etching times of each sample. (c) Variation of residual Ag content in GNPG films by XPS depth profile with 8 etching times of each sample. 
Table 1 Compositions of GNPG films and the corresponding alloy precursors measured by EDS

\begin{tabular}{lll}
\hline & $\mathrm{Au}(\mathrm{at} \%)$ & $\mathrm{Ag}(\mathrm{at} \%)$ \\
\hline $\mathrm{Au}_{15-40} \mathrm{Ag}_{85-60}$ & 36.8 & 63.2 \\
$\mathrm{Au}_{25-40} \mathrm{Ag}_{75-60}$ & 43.9 & 56.1 \\
$\mathrm{GNPG}_{40-15}$ & 74.2 & 25.8 \\
$\mathrm{GNPG}_{40-25}$ & 71.7 & 28.3 \\
\hline
\end{tabular}

precursors, respectively) which is caused by the $\mathrm{Au}$ adhesion layers. The distribution of $\mathrm{Au}$ and $\mathrm{Ag}$ is uniform as shown in the element mapping (see Fig. S1 $\dagger$ ). The composition of fabricated GNPG films was also measured by XPS depth profile (Fig. 2c) and EDS (Table 1). The residual Ag content on the surface is higher than inside because of the higher Ag content on the surface in precursors. The content of residual Ag of GNPG films is higher than conventional NPG films ${ }^{21}$ which should caused by two factors. One is the short corrosion time. The other is the "parting limit" effect in alloy corrosion, which will be discussed in Section 3.2. The typical XPS spectra of $\mathrm{Ag} 3 \mathrm{~d}$ and $\mathrm{Au} 4 \mathrm{f}$ of precursors and GNPG films are provided in Fig. S2. $\dagger$

\subsection{Microstructure of GNPG films}

Fig. 3 shows typical SEM images of the two kinds of GNPG nanostructures. The nanoporous gold like morphologies, i.e., the ligament and pore bicontinuous nanostructure, can be observed in Fig. 3a and b. No cracks or other defects exist in the surface views of the fabricated GNPGs, which indicates an excellent structural homogeneity of these GNPGs in a large area (at least $2 \times 3 \mu \mathrm{m}^{2}$ ). Unlike the low magnification SEM images, a dramatic difference of microstructures can be seen in these two kinds of GNPG nanostructures. As shown in the inset of Fig. $3 \mathrm{~b}$, the microstructure of $\mathrm{GNPG}_{40-25}$ (the GNPG film fabricated by $\mathrm{Au}_{25-40} \mathrm{Ag}_{75-60}$ precursor film) appears a continuous

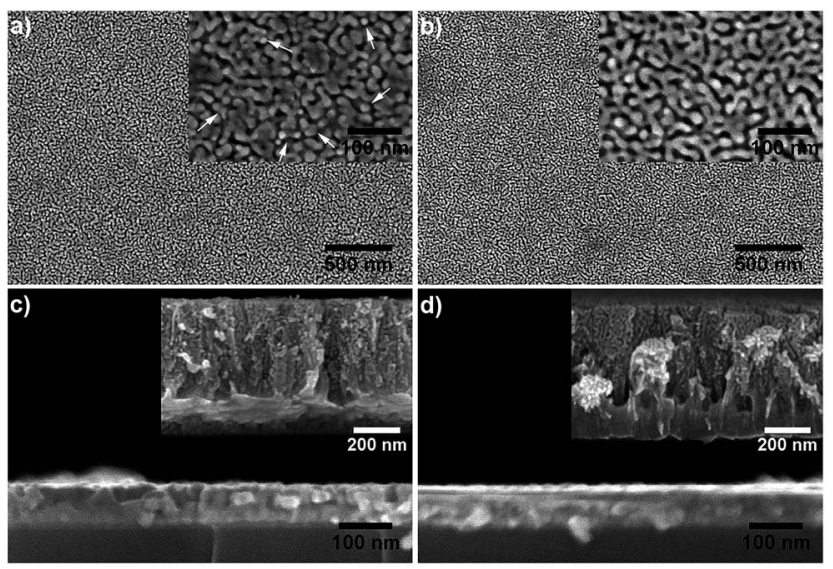

Fig. 3 SEM images of GNPG films. (a) $\mathrm{GNPG}_{40-15}$, surface-view; (b) GNPG $_{40-25}$, surface-view; (c) GNPG $40-15$, section-view; (d) GNPG $40-25$, section-view. Insets of (a) and (b) are high magnification SEM images of the surface-views for corresponding GNPG films. Insets of (c) and (d) are the section-views of thicker ( $550 \mathrm{~nm})$ GNPG films with the same composition of corresponding films. network of branched ligaments in the full range, which is the same as normal NPG films. ${ }^{22}$ While the ligaments of GNPG $_{40-15}$ (the GNPG film fabricated by $\mathrm{Au}_{15-40} \mathrm{Ag}_{85-60}$ precursor film as shown in inset of Fig. 3a) are partially continuous, some isolated islands (where the arrows aim at) can be observed. Moreover, the ligament size of these two GNPGs is nearly equal.

As we know, a "parting limit" exists in the dealloying process, i.e., only alloys within a certain range of compositions can be dealloyed. ${ }^{23}$ Generally, for dealloying AuAg alloy, the continuous ligament structure would collapse and the films tend to fracture or even disintegrate when the content of $\mathrm{Ag}$ is lower than 20 at $\%{ }^{23-25}$ For $\mathrm{GNPG}_{40-15}$, the $\mathrm{Ag}$ content of its precursor $\left(\mathrm{Au}_{15-40} \mathrm{Ag}_{85-60}\right.$ alloy) is lower than 20 at\% in the top surface range with the thickness of $\sim 20 \mathrm{~nm}$. Thus, the ligament structure in the top range of $\mathrm{GNPG}_{40-15}$ tends to collapse during the dealloying process. Observation on $\mathrm{GNPG}_{40-15}$ films shows that there is neither disintegration nor cracks in the full surface (Fig. 3a), which indicates the successful fabrication of a NPG structure from an alloy precursor with $\mathrm{Au}$ content as low as 15 at\%.

This result should attribute to the gradient composition of GNPG precursors, which can lead to a NPG structure with the gradient distribution of ligaments and pores. The formation of nanoporous gold structure through dealloying AuAg alloy is controlled by two mean physical processes: the dissolution of silver atoms into the electrolyte and the coalescence of gold adatoms into stable clusters. ${ }^{6,26}$ The dissolution of silver atoms generally initiates from the grain boundaries of the alloy surface, then spread throughout the entire alloy layer for the increasing interfacial boundary energy in grain boundary areas, which making them more susceptible to chemical attack..$^{11,27}$ Clearly, the dissolution of silver atoms has no influence on the distribution of remaining gold atoms, i.e., the distribution of ligaments and pores. The coalescence or diffusion of gold adatoms is the crucial factor of the final dealloying structure. According to the kinetic Monte Carlo simulation by Erlebacher J. et al., ${ }^{6}$ the diffusion of gold adatoms is confined to the interface region between the alloy and the electrolyte. Thus, the diffusion of gold only has limited influence on the distribution of gold atoms. Overall, the gradient distribution of gold atoms would not be damaged during the dealloying process, which could lead to a nanoporous gold structure with gradient distribution of ligaments and pores, i.e. GNPG.

To confirm the above supposition, the cross-sectional SEM images of GNPGs are provided in Fig. $3 \mathrm{c}$ and d. The film thickness was reduced from $\sim 90 \mathrm{~nm}$ to $\sim 70 \mathrm{~nm}$ due to the shrinkage during selective etching. ${ }^{28}$ It is hardly to observe the subtle microstructure from the cross-section of fabricated GNPGs due to the extremely thin layer and small ligament size. Therefore, thicker GNGP films $(\sim 550 \mathrm{~nm})$ with the same composition were fabricated for the section view exploration. The observation on these cross-section images (as shown in insets of Fig. $3 \mathrm{c}$ and d) confirms that the ligament and pore structure was formed throughout the entire layer with a looser structure on the top and a denser structure at the bottom, which represents the variation of porosities along the thickness direction. It should be noted that no visible pores could be seen 
at the bottom of these GNPG layers, which indicates that the etching is weak. The content of $\mathrm{Au}$ on the bottom is $\sim 40$ at $\%$ which exceeds the "parting limit", thus the etching becomes hard. The increase of residual Ag after 150 s etching in XPS depth profile (Fig. 2c) is another evidence of this deduction. The incomplete etching of GNPGs also leads to a large Ag residual (see the results of EDS in Table 1). The results above demonstrate that a complex ligament and pore structure exists in the thickness direction of GNPG films. This complex nanostructure can bring abundant inner structures into the dull NPG structure, which should thus produce excellent properties in GNPG films.

\subsection{SERS of GNPG films}

The SERS measurements were implemented via using R6G as the probe molecule. Different Au content in alloy precursors will lead to different porosities in the final dealloyed nanoporous gold, which would significantly affect the SERS property of NPG films., ${ }^{9,12}$ Thus, two NPG films with different Au content were used as contrast. As shown in Fig. 4a, the GNPG films lead to obvious improvement in the SERS enhancements in comparison with the conventional NPG films. The Raman intensity of GNPG films is about 3-5-fold stronger than NPG films (see Table 2), suggests that GNPG films should be a more potential SERS substrate than NPG films. More interestingly, the variation of Raman intensity for these two kinds of nanoporous gold is quite different. For NPG films, the Raman intensity of $\mathrm{NPG}_{25}$ (the NPG film fabricated by $\mathrm{Au}_{25} \mathrm{Ag}_{75}$ ) is $\sim 1.5$-fold stronger than that of $\mathrm{NPG}_{15}$ (the NPG film fabricated by $\mathrm{Au}_{15} \mathrm{Ag}_{85}$ ). On the contrary, for GNPG films, the Raman intensity of $\mathrm{GNPG}_{40-15}$ is $\sim 1.7$-fold stronger than $\mathrm{GNPG}_{40-25}$. This result implies that the enhancement mechanisms of these two kinds of nanoporous structures should
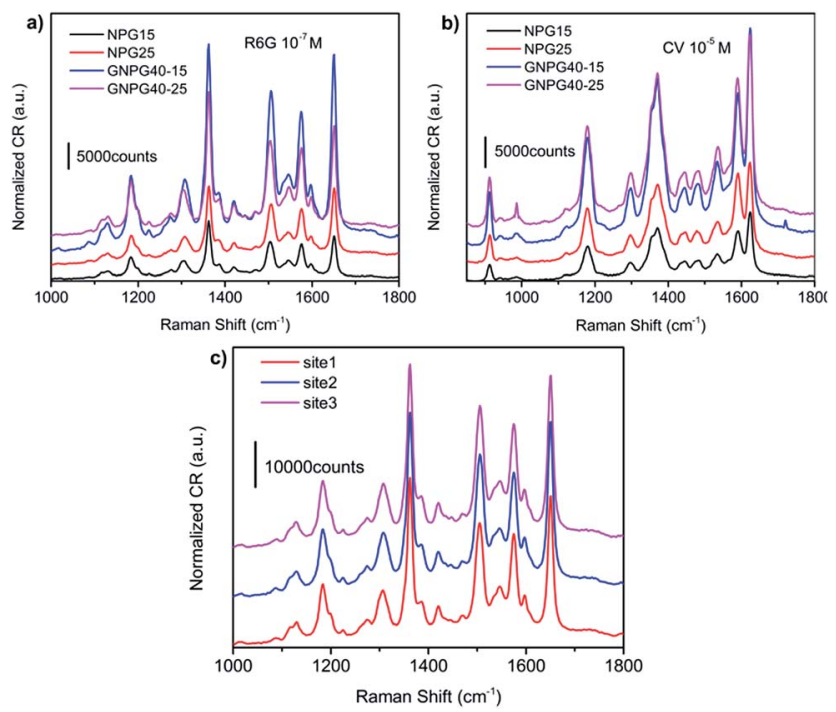

Fig. 4 SERS spectra of GNPG films and NPG films fabricated by different precursors. (a) and (b) are the comparison of SERS spectra between GNPG films and NPG films with $10^{-7} \mathrm{M} \mathrm{R6G}$ and $10^{-5} \mathrm{M} \mathrm{CV}$ as the probe molecules, respectively. (c) Variation of SERS spectra of $10^{-7}$ M R6G on GNPG $40-15$ probed at different sites. The excitation wavelength is $514.5 \mathrm{~nm}$ for both molecules.
Table 2 The relative intensity of the two main Raman bands of R6G for different samples. The intensity was taken as the integral area of Raman bands

\begin{tabular}{lll}
\hline & \multicolumn{2}{l}{ Relative intensity $(I)$} \\
\cline { 2 - 3 } & $1363 \mathrm{~cm}^{-1}$ & $1651 \mathrm{~cm}^{-1}$ \\
\hline $\mathrm{GNPG}_{40-15}$ & $742063.3 \pm 27000$ & $711140.1 \pm 23000$ \\
$\mathrm{GNPG}_{40-25}$ & $481424.7 \pm 19000$ & $363469.1 \pm 17000$ \\
$\mathrm{NPG}_{15}$ & $193514.3 \pm 9500$ & $134757.3 \pm 6600$ \\
$\mathrm{NPG}_{25}$ & $241210.3 \pm 11000$ & $244804.1 \pm 10000$
\end{tabular}

be different, which will be discussed later. To confirm the generality of these results, the SERS spectra of CV molecule were also measured as shown in Fig. 4b. Similar results can be obtained from Fig. 4b, which indicates that it is the inherent property for GNPG films to possess more novel SERS features.

The SERS homogeneities of GNPG films were also investigated by acquiring the Raman signals from three different sites of GNPG $_{40-15}$. As shown in Fig. 4c, the Raman intensity of these three sites is approximately equal, which indicates that GNPG films are homogeneous enhanced SERS substrates. The homogeneous Raman enhancement of GNPGs originates from their extremely homogeneous distribution of ligaments and pores as shown in Fig. 3a and b.

To precisely describe the SERS properties, the relative enhancement factor $\left(\mathrm{EF}_{\mathrm{R}}\right)$ of GNPG films versus NPG films was estimated by the following equation: ${ }^{14,29}$

$$
\mathrm{EF}_{\mathrm{R}}=\left(I_{\mathrm{GNPG}} / N_{\mathrm{GNPG}}\right) /\left(I_{\mathrm{NPG}} / N_{\mathrm{NPG}}\right)
$$

where $I_{\mathrm{GNPG}}$ and $N_{\mathrm{GNPG}}$ are the intensity of the SERS signal and the number of probe molecules contributing to the Raman signal of GNPG films, respectively. $I_{\mathrm{NPG}}$ and $N_{\mathrm{NPG}}$ are corresponding parameters of NPG films. To discuss easily, eqn (1) is rewritten to the following form:

$$
\mathrm{EF}_{\mathrm{R}}=\left(I_{\mathrm{GNPG}} / I_{\mathrm{NPG}}\right) \times\left(N_{\mathrm{NPG}} / N_{\mathrm{GNPG}}\right)
$$

where $I_{\mathrm{GNPG}} / I_{\mathrm{NPG}}$ can be gotten easily from Table 2 . Nevertheless, it is hard to estimate the value of $N_{\mathrm{NPG}} / N_{\mathrm{GNPG}}$ for the adsorption of the probed molecules on the substrates is not quite clear. If the adsorption of probed molecules is assumed to be uniform (considering the extremely dilute solution of probe molecule, this assumption is reasonable), $N_{\mathrm{NPG}} / N_{\mathrm{GNPG}}$ should only be related to the effective surface area ( $\left.A_{\text {eff }}\right)$, which has contributions to the Raman signal, of these two kinds of substrates. It is known that, for Raman measurement, the laser beam generally has a penetration depth $(D)$. If the influence of the attenuation of laser on Raman signal is assumed to ignore, $A_{\text {eff }}$ should be proportional to the volume exposed by the laser beam, i.e., $A_{\text {eff }}=\alpha_{\mathrm{v}} D A_{\text {spot }}$, where $\alpha_{\mathrm{V}}$ and $A_{\text {spot }}$ are the total surface area in per unit volume and the laser spot size, respectively (see Fig. 5a). Considering $D$ is usually over several micrometers, ${ }^{17,29}$ which is much thicker than the thickness of films ( $D>h$, as shown in Fig. 5b), therefore the assumption above is reasonable. $A_{\text {eff }}$ could be estimated via $A_{\text {eff }}=\alpha_{\mathrm{v}} h A_{\text {spot }}$. 


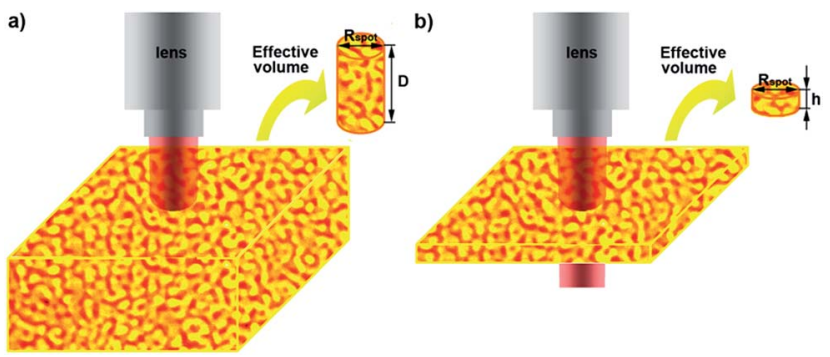

Fig. 5 Schematic diagram of SERS measurement with different film thickness, $h$. (a) $h>D$. (b) $h<D$, where $D$ is the penetration depth of laser beam. The effective volume represents the volume in the samples that has contributions to the Raman signal. $R_{\text {spot }}$ is the radius of laser spot.

Therefore, $N_{\mathrm{NPG}} / N_{\mathrm{GNPG}}$ can be estimated from the following equation:

$$
N_{\mathrm{NPG}} / N_{\mathrm{GNPG}}=A_{\mathrm{eff}_{\mathrm{NPG}}} / A_{\mathrm{eff}_{\mathrm{GNPG}}}=\alpha_{\mathrm{V}_{\mathrm{NPG}}} / \alpha_{\mathrm{V}_{\mathrm{GNPG}}}
$$

note $h$ and $A_{\text {spot }}$ are the same for all samples. According to its definition, $\alpha_{\mathrm{v}}$ can be easily estimated via $\alpha_{\mathrm{v}}=A_{\text {net }} / A_{\mathrm{ex}} h$, where $A_{\text {net }}$ and $A_{\text {ex }}$ is the net surface area and external surface area of nanoporous gold samples, respectively. $A_{\text {net }}$ can be measured by means of the electrochemical capacitance ratio method..$^{20,30}$ The net surface area was taken to obey $A_{\text {net }}=I / \dot{E} c_{\mathrm{dl}}$, where $c_{\mathrm{dl}}$ is the capacitance value of $\mathrm{Au}$ in the double-layer region of the voltammogram. $A_{\text {ex }}$ was taken as the contact area of working electrode with electrolyte of measured samples $\left(1 \mathrm{~cm}^{2}\right)$. Fig. $6 \mathrm{a}$ shows the cyclic voltammograms (CVs) of $\mathrm{GNPG}_{40-15}$ and $\mathrm{NPG}_{15}$ in the double-layer region at different scan rates. The averages of the capacitive currents, $I_{\mathrm{c}}$, taken when $E=0.25 \mathrm{~V}$, are shown in Fig. 6b. Based on the measurements above, $\mathrm{EF}_{\mathrm{R}}$ of $\mathrm{GNPG}_{40-15}$ versus $\mathrm{NPG}_{15}$ is estimated to be $\sim 8$. Considering the enhancement factor of NPGs is over $10^{6}$ magnitudes, the EF of GNPGs should over $10^{7}$. More remarkably, the process to fabricate gradient nanoporous gold films is highly compatible with other NPG nanostructure preparing strategies. For example, it is easy to fabricate wrinkled GNPG films following the method proposed by Zhang L. et al. ${ }^{14}$ It is predicted that the GNPG nanostructures with the EF over $10^{10}$ can be fabricated by combining our strategy with other preparing strategies. This
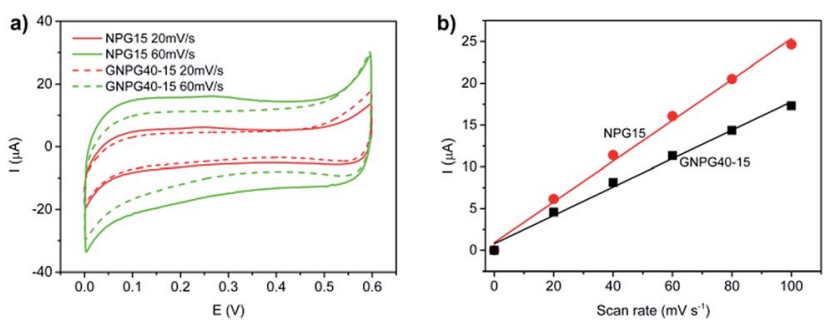

Fig. 6 Electrochemical characterization of gradient nanoporous gold (GNPG) and nanoporous gold (NPG). (a) Cyclic voltammograms of current I versus electrode potential $E$ in the nominally capacitive regime using different scan rates. (b) Mean current magnitude, $I_{c}$, at $E$ $=0.25 \mathrm{~V}$ versus the scan rate. could be an important improvement for single molecule detection.

The enhancement mechanisms of GNPG films are discussed below. According to Zhang L. et al. ${ }^{14}$ if the SERS enhancement originates from the chemical effect, it will lead to the Raman band shift of $\sim 20 \mathrm{~cm}^{-1}$ and a stronger enhancement of depolarized bands (dp) than polarized bands (p). Three pairs of Raman bands of the $\mathrm{CV}$ molecules are investigated as shown in Fig. 7. Clearly, the Raman band shifts of these peaks compared with that in the FT-Raman spectra ${ }^{31}$ are less than $20 \mathrm{~cm}^{-1}$ and the Raman intensities of dp modes are weaker than $\mathrm{p}$ modes. Thus, the chemical effect should have negligible influence on the Raman enhancement of GNPG films.

Silver may have significant contributions for the enhanced Raman signal of GNPGs because of its stronger SERS enhancement than gold. ${ }^{32}$ According to the results of XPS (Fig. 2c) and EDS (Table 1), the content of residual silver in $\mathrm{GNPG}_{40-15}$ is less than $\mathrm{GNPG}_{40-25}$. Therefore, if the residual silver in GNPG films plays a key role in the SERS enhancement, the Raman intensity of $\mathrm{GNPG}_{40-25}$ should be stronger than that of $\mathrm{GNPG}_{40-15}$. However, the results of Raman spectra are just opposite. Thus, the residual silver in GNPGs is not the major effect on the Raman enhancement.

For NPG films, like other gold nano structures, the free electrons in nanosized gold ligaments is believed to generate localized surface plasmon resonance (LSPR) when excited by appropriate incident light, which can enhance the electromagnetic field around the ligaments. ${ }^{12,33}$ Generally, the enhancement of electromagnetic field is finite for isolated nano structures. While for NPG films, their unique bi-continuous structures can generate intense near-field coupling effect between the neighbouring ligaments, then leads to tremendous electromagnetic enhancement. ${ }^{9,12,34}$ For GNPG films, on the one hand, they have the similar bi-continuous ligament and pore structures to NPG films, thus it is no doubt that the LSPR effect and near-field coupling effect play a key role on the SERS of GNPGs. On the other hand, the unique gradient structure of GNPGs should have non negligible effect, we called it gradient effect, on their SERS properties. Clearly, the Raman intensity of $\mathrm{GNPG}_{40-15}$ is $\sim 1.7$-fold stronger than $\mathrm{GNPG}_{40-25}$, which should be caused by the increased gradient effect of $\mathrm{GNPG}_{40-15}$. It is no doubt that the gradient effect will increase with the increase of structural gradient. To further prove the existence of gradient
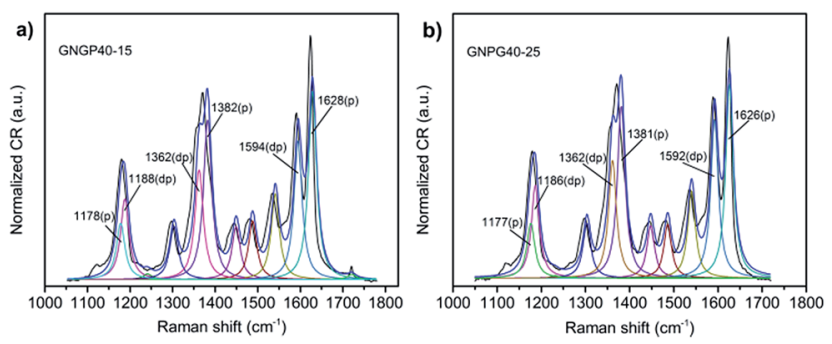

Fig. 7 Peak fits of SERS spectra for different GNPG films. (a) GNPG $40-15$. (b) GNPG $40-25$. The three pairs of Raman bands were labeled for the comparison of Raman band shifts with the FT-Raman spectra. 

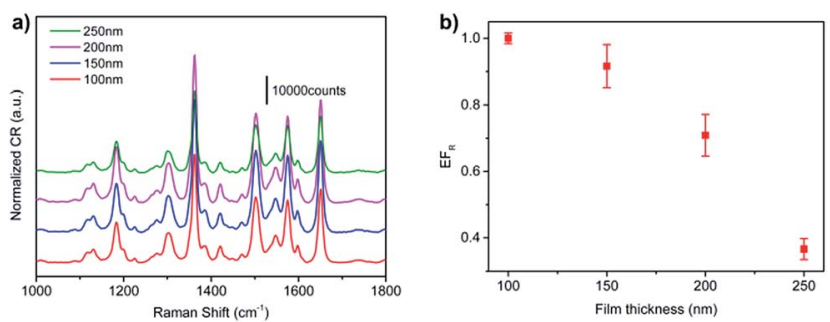

Fig. 8 SERS results of GNPG $_{40-15}$ films with the thickness of $100 \mathrm{~nm}$, $150 \mathrm{~nm}, 200 \mathrm{~nm}$ and $250 \mathrm{~nm}$, respectively. (a) SERS spectra using R6G as the probe molecules. (b) Variation of $E F_{R}$ with film thickness.

effect, the SERS properties of $\mathrm{GNPG}_{40-15}$ films with different thickness are discussed for the increase of film thickness will decrease the structural gradient. Fig. 8a shows the SERS spectra of these samples. To intuitively compare their SERS effect, the $\mathrm{EF}_{\mathrm{R}}$ is thus estimated as shown in Fig. 8b (the corresponding cyclic voltammograms results are provided in Fig. $\mathrm{S} 3 \uparrow$ ). $T$ he $\mathrm{EF}_{\mathrm{R}}$ of $\mathrm{GNPG}_{40-15}$ distinctly decreases with the increase of film thickness, which dramatically coincides with the decrease of structural gradient. Therefore, the gradient effect should play a key role in the SERS properties of GNPG films. We believe that for GNPG films, their SERS enhancement is the combination of the LSPR effect of nanosized gold ligaments, the near-field coupling effect of neighbouring ligaments and the gradient effect of the gradient structures. We think the gradient effect may be a kind of structural coupling effect along the thickness direction, which could further enhance the local electric field compared to conventional NPGs. Moreover, this kind of structural coupling effect could easily be increased by the increase of structural gradient. The detailed physical mechanism will be the topic of our further work.

\section{Conclusions}

We have developed a novel gradient structural nanoporous gold substrate with high Raman enhancement effect, excellent SERS homogeneity and wonderful processing compatibility by combining the traditional magnetron sputtering with dealloying technique. The gradient composition of dealloying precursors leads to the gradient structure of dealloying products, which turns the monotonous NPG films to a vertical 3D nanostructure with abundant inner structures. The gradient structure of GNPG films plays a key role on their SERS enhancement. The relative enhancement factor of GNPG films versus NPG films is estimated to be $\sim 8$ via the calculation of effective surface area. It is believed that the tremendous Raman enhancement of GNPG films is caused by the combination of the LSPR effect of nanosized gold ligaments, the near-field coupling effect of neighbouring ligaments and the gradient effect of the gradient structures.

\section{Acknowledgements}

This research was supported by the National Natural Science Foundation of China (Grant No. 51401194).

\section{Notes and references}

1 C. L. Haynes, A. D. McFarland and R. P. V. Duyne, Anal. Chem., 2005, 338A-346A.

2 J. P. Camden, J. A. Dieringer, J. Zhao and R. P. V. Duyne, Acc. Chem. Res., 2008, 41, 1653-1661.

3 S. O. Kucheyev, J. R. Hayes, J. Biener, T. Huser, C. E. Talley and A. V. Hamza, Appl. Phys. Lett., 2006, 89, 053102.

4 M. Fleischmann, P. J. Hendra and A. J. Mcquillan, Chem. Phys. Lett., 1974, 26, 163-166.

5 Y. Zhong, J. Markmann, H.-J. Jin, Y. Ivanisenko, L. Kurmanaeva and J. Weissmuller, Adv. Eng. Mater., 2014, 16, 389-398.

6 J. Erlebacher, M. J. Aziz, A. Karma, N. Dimitrov and K. Sieradzki, Nature, 2001, 410, 450-453.

7 Y. Ding, Y.-J. Kim and J. Erlebacher, Adv. Mater., 2004, 16, 1897-1900.

8 J. Biener, G. W. Nyce, A. M. Hodge, M. M. Biener, A. V. Hamza and S. A. Maier, Adv. Mater., 2008, 20, 1211-1217.

9 L. H. Qian, X. Q. Yan, T. Fujita, A. Inoue and M. W. Chen, Appl. Phys. Lett., 2007, 90, 153120.

10 Q. Zhang, X. Wang, Z. Qi, Y. Wang and Z. Zhang, Electrochim. Acta, 2009, 54, 6190-6198.

11 R. Morrish, K. Dorame and A. J. Muscat, Scr. Mater., 2011, 64, 856-859.

12 X. Y. Lang, L. Y. Chen, P. F. Guan, T. Fujita and M. W. Chen, Appl. Phys. Lett., 2009, 94, 213109.

13 Y. Jiao, J. D. Ryckman, P. N. Ciesielski, C. A. Escobar, G. K. Jennings and S. M. Weiss, Nanotechnology, 2011, 22, 295302.

14 L. Zhang, X. Lang, A. Hirata and M. Chen, ACS Nano, 2011, 5, 4407-4413.

15 F. Zhao, J. Zeng, M. M. Parvez Arnob, P. Sun, J. Qi, P. Motwani, M. Gheewala, C. H. Li, A. Paterson, U. Strych, B. Raja, R. C. Willson, J. C. Wolfe, T. R. Lee and W. C. Shih, Nanoscale, 2014, 6, 8199-8207.

16 J. Zeng, F. Zhao, J. Qi, Y. Li, C.-H. Li, Y. Yao, T. R. Lee and W.-C. Shih, RSC Adv., 2014, 4, 36682.

17 J. Qi, P. Motwani, M. Gheewala, C. Brennan, J. C. Wolfe and W. C. Shih, Nanoscale, 2013, 5, 4105-4109.

18 G. Ruffato, D. Garoli, S. Cattarin, S. Barison, M. Natali, P. Canton, A. Benedetti, D. De Salvador and F. Romanato, Microporous Mesoporous Mater., 2012, 163, 153-159.

19 S. Trasatti and O. A. Petrii, Pure Appl. Chem., 1991, 63, 711734.

20 Z. Qi and J. Weissmüller, ACS Nano, 2013, 7, 5948-5954.

21 E.-J. Gwak, N.-R. Kang, U. B. Baek, H. M. Lee, S. H. Nahm and J.-Y. Kim, Scr. Mater., 2013, 69, 720-723.

22 B. H. Rösner, S. Parida, D. Kramer, C. A. Volkert and J. Weissmüller, Adv. Eng. Mater., 2007, 9, 535-541.

23 A. Wittstock, J. Biener and M. Baumer, Phys. Chem. Chem. Phys., 2010, 12, 12919-12930.

24 K. Sieradzki, N. Dimitrov, D. Movrin, C. McCall, N. Vasiljevic and J. Erlebacher, J. Electrochem. Soc., 2002, 149, B370.

25 R. C. Newman, S. G. Corcoran, J. Erlebacher, M. J. Aziz and K. Sieradzki, MRS Bull., 1999, 24-28. 
26 J. Erlebacher, J. Electrochem. Soc., 2004, 151, C614.

27 X. Lu, T. J. Balk, R. Spolenak and E. Arzt, Thin Solid Films, 2007, 515, 7122-7126.

28 S. Parida, D. Kramer, C. Volkert, H. Rösner, J. Erlebacher and J. Weissmüller, Phys. Rev. Lett., 2006, 97, 035504.

29 C. Fang, J. G. Shapter, N. H. Voelcker and A. V. Ellis, RSC $A d v .$, 2014, 4, 19502.

30 S. Trasatti and A. Petrii, Pure Appl. Chem., 1991, 63, 711-734.
31 M. V. Canamares, C. Chenal, R. L. Birke and J. R. Lombardi, J. Phys. Chem. C, 2008, 112, 20295-20300.

32 L. Zhang, L. Chen, H. Liu, Y. Hou, A. Hirata, T. Fujita and M. Chen, J. Phys. Chem. C, 2011, 115, 19583-19587.

33 E. Detsi, M. Salverda, P. R. Onck and J. T. M. De Hosson, J. Appl. Phys., 2014, 115, 044308.

34 L. H. Qian, A. Inoue and M. W. Chen, Appl. Phys. Lett., 2008, 92, 093113. 